\title{
USO DEL SUELO Y SU INFLUENCIA EN LA PRESIÓN Y DEGRADACIÓN DE LOS RECURSOS HIDRICOS EN CUENCAS HIDROGRÁFICAS
}

\author{
USE OF SOIL AND ITS INFLUENCE \\ IN THE PRESSURE AND DEGRADATION OF WATER \\ RESOURCES IN WATERSHEDS
}

\author{
Diego Javier Perez Ortega ${ }^{1}$ \\ Jorge Andrés Segovia Ortega ${ }^{2}$ \\ Paulo Cesar Cabrera Moncayo ${ }^{3}$ \\ Iván Andrés Delgado Vargas 4 \\ Marcelo Luiz Martins Pompêo ${ }^{5}$
}

${ }^{1}$ 1PhD en Ciencias Ambientales, Docente - Departamento de Recursos Naturales

y Sistemas Agroforestales de la Universidad de Nariño - Colombia.

2 Ingeniero Agroindustrial, Facultad de Ingenieria Agroindustrial - Universidad de Nariño.

${ }^{3}$ MSE. Ingenieria Ambiental, Facultad de Ciencias Agrícolas - Universidad de Nariño.

${ }^{4}$ MS.c Agroforestería Tropical, Facultad de Ciencias Agrícolas - Universidad de Nariño.

${ }^{5}$ PhD en Ciencias de la ingeniería Ambiental, Instituto de Biociências - Universidade de São Paulo.

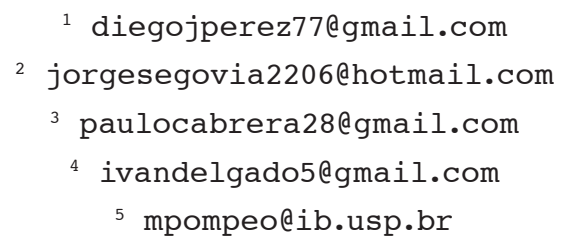

\section{Resumen}

Esta investigación tuvo como objetivo, determinar las relaciones existentes entre el uso de del suelo y la calidad del agua de la cuenca del rio Juqueri, mediante las correlaciones canónicas que existen entre los parámetros que componen el ICA y los cambios en el uso del suelo en los últimos 30 años, esto a partir de imágenes de satélite LANDSAT 5, 7 y 8 mediante la clasificación supervisionada, que determinó 6 clases de uso. Para determinar el ICA en la cuenca se recogieron 8 muestras en el período de verano y 8 en el invierno en los años 2015 y 2016 en 3 
puntos de la cuenca, en su parte alta, media y en su desembocadura. Los resultados obtenidos revelaron que los cuerpos de agua sufrieron una disminución de área que pasó del 1,1\% al 0,68\% disminuyendo en total el 0,42\%. En relación a ICA en algunos puntos muestrales se observaron valores por debajo de 51 que se clasifica como agua de calidad Regular para el consumo humano. Se encontraron correlaciones entre la disminución en los valores de DBO por las áreas urbanas. Ante lo expuesto, se verifica la necesidad, de planificación del uso de suelos y un continuo gerenciamiento del recurso hídrico de la cuenca.

Palabras Clave: Correlación canónica, herramientas estadísticas, indice de calidad de aguas, variable.

\section{Abstract}

The objective of this research was to determine the relationship between soil use and water quality in the Juqueri River basin, through the canonical correlations that exist between the parameters that make up the IQW and the changes in soil use in the last 30 years, this from LANDSAT satellite images 5, 7 and 8 through the supervised classification, which determined 6 classes of use. To determine the IQW in the basin, 8 samples were collected in the summer period and 8 in the winter in 2015 and 2016 in 3 points of the basin, in its upper, middle and at its River mouth. The results obtained revealed that the water bodies suffered a decrease in area from $1.1 \%$ to $0.68 \%$, decreasing a total of $0.42 \%$. In relation to IQW in some sampling points, values were observed below 51, which is classified as Regular quality water for human consumption. Correlations were found between the decrease in BOD values by urban areas. Therefore, the need for planning the use of land and a continuous management of the water resources of the basin was verified.

Key words: Canonical correlation, statistical tools, variable, water quality index.

\section{Introducción}

Los diversos usos del suelo por el hombre alteran los procesos biológicos, físicos y químicos de los sistemas naturales y contribuyen a la reducción de la calidad del agua (Oliveira-Filho, et al., 2012).

Existen características inherentes de una determinada cuenca hidrográfica que puede contribuir o potenciar la degradación ambiental en sus recursos hídricos, de ahí la importancia de determinar mediante índices e indicadores, como los cambios en el uso de la tierra, acompañados de las actividades antrópicas, ejercen una presión ambiental en estos recursos (Pérez-Ortega, et al., 2016).
Partiendo de la hipótesis que la agricultura es la forma más representativa de las actividades antrópicas, pues ejerce cierta influencia en el ciclo hidrológico natural en las cuencas hidrográficas. Esto ocurre, en parte, por utilizar grandes cantidades de agua y contribuye con fuentes difusas de contaminantes (Ribeiro, 2009), además de influir en sus recursos hídricos, tanto en su calidad, cuanto en su cantidad.

Los cambios en la cobertura de suelo, perdiendo capa vegetal, seguramente ocasionan disminución de aportaciones hídricas, debido a su pérdida de capacidad de infiltración y recarga de acuíferos, ocasionando impactos negativos en la disponibilidad de los recursos hídricos en cualquier época del año. 
De esta forma, la estructura temporal que el uso del suelo tiene naturalmente diseñada, se ve deteriorada por las actividades antrópicas, factor que ayuda a la escorrentía, en procesos como la erosión, lo cual degrada la calidad de aguas de esta cuenca, además de crear zonas críticas ocupadas por los sectores agrícola, forestal, urbano y turístico, ayudan en el crecimiento exponencial de la presión y fragilidad del agua de esta cuenca. Siendo así, se pretende identificar, si en la cuenca hidrográfica del río Juqueri la agricultura es la actividad antrópica más impactante en los recursos hídricos.

La calidad del agua de una cuenca hidrográfica puede verse afectada por varios factores antrópicos o naturales, así como la interacción entre ellos (Pérez-Ortega, et al., 2016). Por lo tanto, los valores de referencia que reflejan la calidad del agua deben ser establecidos, preferentemente a través del monitoreo de los parámetros físicos, químicos y biológicos (Haddad, 2007).

En Brasil, la clasificación de los cuerpos de agua superficial en relación a su calidad es establecida por la resolución 357 del Consejo Nacional de Medio Ambiente (CONAMA), sirviendo como soporte indispensable a los programas de gestión de los recursos hídricos (Brasil, 2005).

Por lo tanto, el ICA es un índice de evaluación de la calidad del agua que fue desarrollado en 1970, en los Estados Unidos, por la National Sanitation Foundation. A partir de 1975, comenzó a ser utilizado por la CETESB (Compañía Ambiental del Estado de São Paulo). Se compone de 9 parámetros para evaluar la calidad del agua que ayuda en el monitoreo y la gestión de los recursos hídricos.

El Índice de Calidad del Agua (ICA) es una herramienta para la evaluación de su calidad, más no permite identificar patrones de distribución de las circunstancias geográficas, históricas y antrópicas ejercidas sobre los recursos hídricos y las respuestas a esos factores. Al trabajar en cuencas hidrográficas es necesario identificar su marco climático, geológico e histórico, pues cada cuenca tiene sus particularidades, que puede modificar las variables físicas, químicas y biológicas del agua (Pérez-Ortega, 2016).

EI ICA es una fuente de información importantísima sobre el estado de calidad del agua de un manantial. Una vez determinado, sirve de referencia en la gestión ambiental, medio de divulgación de la calidad del agua a la sociedad civil y un mecanismo de reunión de parámetros en una única variable de fácil divulgación e interpretación (López 2007).

Mediante este índice se buscó identificar patrones de distribución de las circunstancias geográficas, históricas y antrópicas ejercidas sobre los recursos hídricos de la cuenca de estudio y sus respuestas a estos factores.

El embalse Paiva Castro entró en operación en 1974 y es el último reservorio del sistema Cantareira, es el principal manantial para abastecimiento público de la Región Metropolitana de São Paulo (RMSP) (CETESB, 2014).

El sistema Cantareira abastece al $65 \%$ de la población metropolitana de São Paulo, produciendo, por lo tanto, más de la mitad del agua consumida por los 19 millones de habitantes. $Y$ es de donde la importancia de este estudio radica, ya que dentro de la cuenca hidrográfica del río Juqueri, se encuentra localizado este manantial extremadamente importante para la Gran São Paulo.

Debido a la necesidad apremiante de utilizar metodologías estadísticas que determinen las diferentes circunstancias referentes a los cambios en el uso del suelo y cómo influyen en las características de los recursos hídricos, hace 
necesario, utilizar herramientas estadísticas para dicho fin, las cuales ayudan a reducir el universo de innumerables variables e identificando así las variables principales que determinen o modifiquen el impacto de los problemas ambientales en las cuencas hidrográficas (Pérez-Ortega, 2016).

Esta investigación buscó determinar las relaciones existentes entre el uso del suelo y la calidad del agua, mediante las correlaciones existen entre los parámetros que componen el ICA y los cambios en los últimos 30 años en el uso del suelo de la cuenca hidrográfica del río Juqueri, para identificar la presión antrópica que estos cambios ejercen sobre los recursos hídricos en la cuenca.

Para realizar este estudio, fueron necesarias metodologías estadísticas como son las correlaciones canónicas que permitieron verificar identificar cambios en las características físico-químicas y biológicas de los recursos hídricos, determinando la relación o dependencia que existe entre las variables en el uso de la tierra que interviene en las variables de la variable calidad del agua (Downing, 2004).

\section{Materiales y métodos}

\section{Localización de la Cuenca del Juqueri en el municipio de Mairiporã-SP}

La cuenca del río Juqueri se encuentra ubicada en las coordenadas geográficas $23^{\circ} 21^{\prime} 25^{\prime \prime}$ $S$ y $45^{\circ} 52^{\prime} 40^{\prime \prime}$ W. La mayoría de su área se encuentra en el municipio de Mairiporã, en menor área se encuentra también en los municipios Atibaia, Nazaré Paulista, Guarulhos, Caieiras y Franco da Rocha (EMPLASA, 2000). El área total de la Cuenca del río Juqueri cuenta con $307,0 \mathrm{~km}^{2}$ de los cuales $114 \mathrm{Km}^{2}$ representan el área urbana, siendo el $80,1 \%$ del total se sitúa dentro del área de la Ley de Protección a los Manantiales (EMPLASA, 2000) (Figura 1).

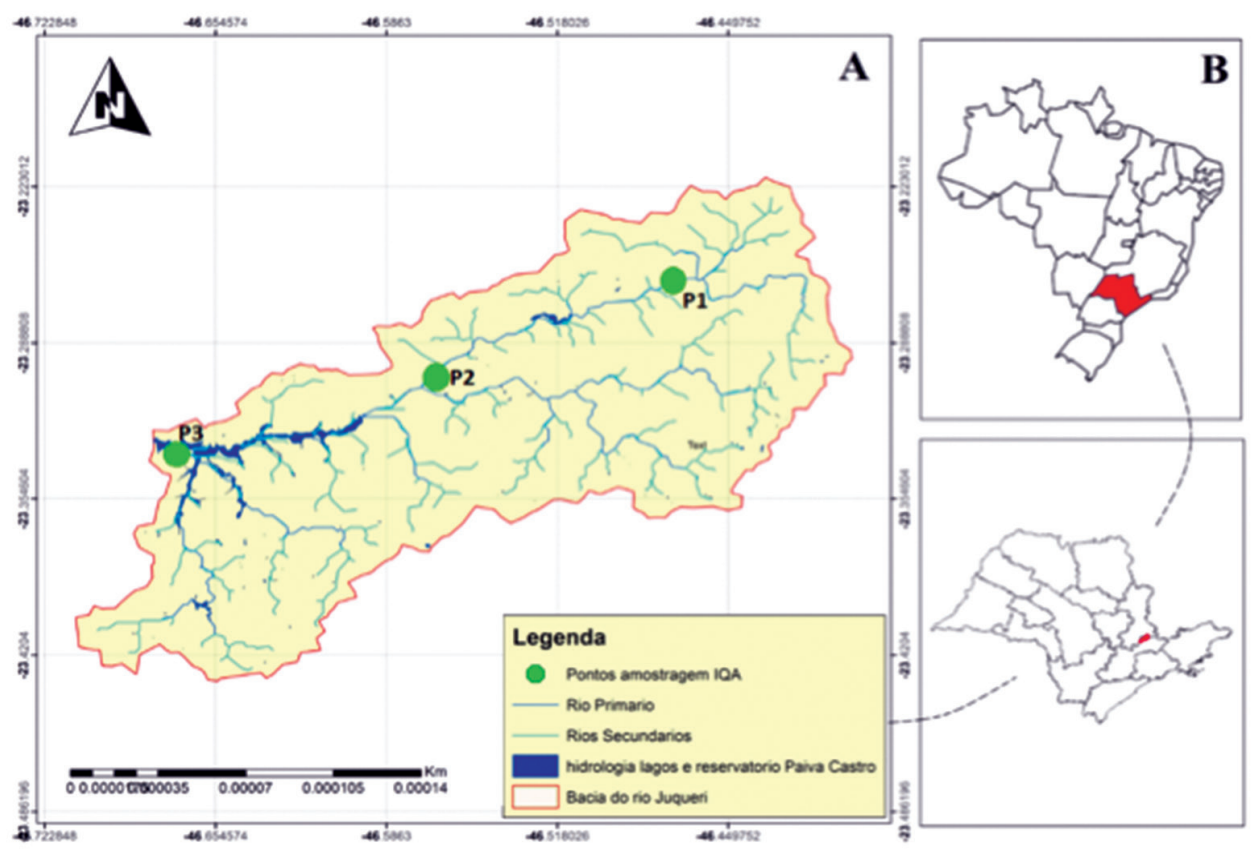

Figura 1. Localización de la Cuenca del río Juqueri - puntos de muestreo, (B) Estado de São Paulo en Brasil. (A) Cuenca del Rio Juqueri en el Estado de São Paulo 


\section{Caracterización del uso y ocupación} del suelo en la Cuenca del río Juqueri

Para la elaboración de los mapas de uso de suelos de los años 1986, 1992, 2003, 2011 y 2016, se realizaron combinaciones de 3 a 8 bandas multiespectrales obtenidas de los satélites Landsat 5, 7 y 8 con sus respectivas firmas espectrales de clases de cobertura obtenidas en la web del SERVICIO GEOLÓGICO DE LOS ESTADOS UNIDOS (USGS). Para la clasificación del área estudiada, se utilizó un método de interpretación en el Programa ArcGIS 10.3.

La interpolación en el Software ArcGis 10.3, predice valores para las celdas de un ráster a partir de una cantidad limitada de puntos de datos de muestra. Puede utilizarse para predecir valores desconocidos de cualquier dato de un punto geográfico, tales como: elevación, precipitaciones, concentraciones químicas, niveles de ruido, etc.

Este método se encuentra en la herramienta Spatial Analyst, con varios métodos de interpolación disponibles, siendo: La herramienta IDW (ponderación de distancia inversa), kriging, vecino natural, spline, spline con barreras, de topo a ráster y la tendencia de interpolación polinómica global (Martínez, 2013).

\section{Índice de calidad del agua (ICA) Muestreo de aguas superficiales}

Este monitoreo proporciono información sobre la calidad natural de las aguas superficiales, suministrando valores de referencia para la represa Paiva Castro. En general, se realiza el primer muestreo de aguas en las nacientes de los ríos, para así obtener una línea base sobre la pureza del agua a ser estudiada, posteriormente se realizan los análisis en los puntos en el centro de la cuenca para identificar las modificaciones temporales y al final de la cuenca, aguas abajo, para conocer los niveles de contaminación que llegan a ser depositados en sus recursos hídricos.
En el presente trabajo se analizaron los resultados obtenidos de las aguas del embalse Paiva Castro, para determinar los parámetros (Oxígeno disuelto, pH, Temperatura, Turbidez, $\mathrm{DBO}_{5}$, Sólidos Totales, Nitrógeno Total, Fosforo Total y Coliformes termotolerantes), dando como resultado un valor referencia y la información global que compone el ICA, demostrando la influencia ejercida negativamente en el saneamiento del agua (Tablas 1 y 2).

Tabla 1. Parámetros de Calidad da agua ICA y respectivos pesos

\begin{tabular}{l|c}
\hline Parâmetro & Peso (w) \\
\hline Oxigeno disuelto & 0,17 \\
\hline Coliformes termotolerantes & 0,15 \\
\hline Potencial hidrogenionico - pH & 0,12 \\
\hline Demanda Bioquímica Oxigeno - DBO5,20 & 0,10 \\
\hline Temperatura & 0,10 \\
\hline Nitrogeno total & 0,10 \\
\hline Fosforo total & 0,10 \\
\hline Turbidez & 0,08 \\
\hline Sólidos totales & 0,08 \\
\hline
\end{tabular}

Fuente: ANA, 2004

Tabla 2. Clasificación de valores ICA

\begin{tabular}{l|c}
\hline Valores & Evaluación ICA \\
\hline $80-100$ & Optima \\
\hline $52-79$ & Buena \\
\hline $37-51$ & Razonable \\
\hline $20-36$ & mala \\
\hline $0-19$ & Pesima \\
\hline
\end{tabular}

Fuente: ANA, 2004

\section{Puntos de muestreo}

Estos fueron elegidos por las siguientes condiciones:

- La facilidad para el acceso con los equipos (turbidímetro, oxímetro, pH y termómetro) para hacer las respectivas lecturas. 
- Se realizaron 3 colectas en la parte alta y media en el curso del río y en la parte baja de la cuenca en el embalse Paiva castro.

- Las muestras fueron recolectadas en el período de la mañana a las 8:00 am (Tabla 3).
- Las muestras se tomaron en los meses de Julio y Diciembre de los años 2015 y 2016, siendo una muestra semanal en total 4 muestras por mes 8 por año, buscando caracterizar los períodos de sequía y de precipitación.

Tabla 3. Coordenadas de los puntos de muestreo para el ICA

\begin{tabular}{l|c|c|c}
\hline \multirow{2}{*}{ Coordenadas } & \multicolumn{3}{c}{ Aguas Superficiales } \\
\hline Latitud & Punto 1 & Punto 2 & Punto 3 \\
\hline Longitud & $23^{\circ} 15^{\prime} 54.51^{\prime \prime} \mathrm{S}$ & $23^{\circ} 18^{\prime} 53.59^{\prime \prime} \mathrm{S}$ & $23^{\circ} 19^{\prime} 51.9^{\prime \prime} \mathrm{S}$ \\
\hline
\end{tabular}

Selección de parámetros y metodologías los métodos estándar para la exploración de agua analíticas para aguas superficiales

Para realizar los análisis de laboratorio y deter- incluye el método espectrofotométrico y lo desminar la calidad del agua superficial, se utilizaron criptivo de los análisis de laboratorio (Tabla 4).

Tabla 4. Síntesis de los métodos y equipos empleados para análisis Físicos - Químicos y Microbiológicos y Límites de Detección. Variables para ser analizadas en las muestras de la cuenca del Juqueri

\begin{tabular}{|c|c|c|c|}
\hline Variable & Método & Limite de Detección & Equipos y Materiales \\
\hline Turbidez (NTU) & Nefelométrico & 0,01 & Turbidímetro/Hach/2100ANv1.2 \\
\hline Color (uH) & Espectrofotométrico & 1 & Espectrofotometro Odyssey/Hach/DR-2500 \\
\hline Temperatura $\left({ }^{\circ} \mathrm{C}\right)$ & Eletrométrico & 0,1 & pHmetro de membrana/Hanna/HI8314 \\
\hline $\mathrm{pH}$ & Eletrométrico & 0,01 & pHmetro de membrana/Hanna/HI8314 \\
\hline $\begin{array}{l}\text { NitrogenoTotal } \\
(\mathrm{mg} \mathrm{l-1} \mathrm{N})\end{array}$ & $\begin{array}{l}\text { Digestión por Persulfato } \\
\text { y Espectrofotométrico }\end{array}$ & 0,1 & $\begin{array}{l}\text { 1. COD Reactor/ Hach } \\
\text { 2. Espectrofotometro Odyssey/ } \\
\text { Hach/DR-2500 }\end{array}$ \\
\hline$(\mathrm{mg} \mathrm{l}-1 \mathrm{P})$ & $\begin{array}{l}\text { PhosVer } 3 \text {, } \\
\text { Digestión por. Acido } \\
\text { Persulfato y } \\
\text { Espectrofotométrico }\end{array}$ & 0,01 & $\begin{array}{l}\text { 1. COD Reactor/ Hach } \\
\text { 2. Espectrofotometro Odyssey/ } \\
\text { Hach/DR-2500 }\end{array}$ \\
\hline $\mathrm{OD}\left(\mathrm{mg} \mathrm{l}^{-1}\right)$ & $\begin{array}{l}\text { Método de Winkler } \\
\text { Modificado }\end{array}$ & 0,1 & Oxímetro \\
\hline DBO & $\begin{array}{l}\text { Método de Diluciones, } \\
\text { Incubado a } 20^{\circ} \mathrm{C}, 5 \text { días }\end{array}$ & 0,1 & Oxímetro \\
\hline Sólidos Totales & Gravimétrico & 1,0 & $\begin{array}{l}\text { 1. Cápsula de Porcelana } \\
\text { 3. Balanza electrónica de } \\
\text { Precisión de } 0,1 \mu \mathrm{g} / \mathrm{Bel} \text { Mark/U210A } \\
\text { 3. Estufa/Marconi/MA033/ } \\
\text { temp. } 120^{\circ} \mathrm{C} \\
\text { 4. Disecador/Pyrex/200mm }\end{array}$ \\
\hline $\begin{array}{l}\text { Coliformes } \\
\text { termotolerantes } \\
\left(\mathrm{NMP} \mathrm{I}^{-1}\right)\end{array}$ & $\begin{array}{l}\text { Contaje de Escherichia } \\
\text { Coli }\end{array}$ & 1,0 & $\begin{array}{l}\text { 1. Método colilert. } \\
\text { Test simples de } 24 \text { horas para coliformes } \\
\text { totales y E. coli }\end{array}$ \\
\hline
\end{tabular}


Descripción de la metodología para la caracterización del uso del suelo de la cuenca hidrográfica del río Juqueri.

La Metodología adoptada fue del IDEAM, (2010) diseñada para la cartografía de los recursos naturales como: el suelo, el clima, la vegetación y usos del suelo, entre otros. Esta metodología es ideal para caracterizar la cobertura y uso actual de la tierra a escalas de: 1: 10.000, 1: 25: 000, y 1: 100.000 junto con la metodología CORINE Land Cover, homologada para Colombia y Brasil, realizada a partir de la interpretación visual de imágenes de satélite del sensor Landsat.

Pasos de la metodología caracterización del uso del suelo:

- Obtención de imágenes por satélite

- Clasificación de imágenes adquiridas de buena calidad

- Reglas de clasificación del método "Máximum Likelihood Classification".

- Validación de la clasificación en campo

\section{Obtención de imágenes por satélite}

Las imágenes adquiridas en esta investigación recibieron un tratamiento realizado por el Servicio Geológico de Estados Unidos (USGS), con una corrección estándar del terreno, nivel 1T, en lo que se refiere a la precisión radiométrica y geométrica sistemática, utilizando los modelos digitales de elevación SRTM, NED, CDAD, DTED, y GTOPO 30, para precisión y corrección topográfica.

Para la elaboración de los mapas de uso del suelo de los años 1986, 1992, 2003, 2011 y 2016, se realizaron combinaciones de 3 a 8 bandas multiespectrales obtenidas de los satélites Landsat 5, 7 y 8 con sus respectivas firmas espectrales de las clases de cobertura de la tierra del sitio USGS.

\section{Reglas de clasificación del método "Máxi- mum Likelihood Classification".}

Existe un 1\% de error al comparar las diferentes imágenes, por su resolución, otra diferencia notable es la reflectancia en las bandas correspondientes que cada sensor produce, de ahí que la clasificación visual (supervisionada) juega un papel muy importante a la hora de la clasificación del uso del suelo para cada imagen, pues por medio de técnicas de interpretación visual se generaron los resultados buscando la mayor veracidad en la clasificación final.

El método utilizado fue el "Iso Cluster" que utilizó el algoritmo de agrupación de Isodatos para determinar las características de las agrupaciones naturales de las celdas en el espacio de atributo multidimensional y almacenó los resultados en un archivo de firma ASCII de salida para posteriormente realizar la clasificación por el método "Máximum Likelihood Classification".

\section{Validación de la clasificación en campo}

Para la validación del método en esta investigación, se realizaron visitas de campo para identificar con exactitud las clasificaciones de los diferentes usos resultantes del procesamiento digital en el software ArcGIS 10.3 obtenidos.

\section{Análisis estadístico}

Se realizaron los análisis de correlaciones canónicas de los resultados obtenidos del ICA en la cuenca del río Juqueri y del uso del suelo, siendo las variables dependientes (parámetros ICA) y las independientes las modificaciones espaciales temporales ejercidas en la cuenca.

Para identificar las variables que determinaron la variabilidad en la calidad del agua en la cuenca, se realizó un análisis estadístico descriptivo multivariado, utilizando el software InfoStat Professional, Past 3.0, IBM SPSS Statistics y CANOCO 4,5 para Windows 8 . 
Se elaboraron matrices de correlación para encontrar la covarianza de cada par de variables medidas. En este caso, el coeficiente de correlación muestral representó la covarianza de los valores muestrales estandardizados, asumiendo valores en el intervalo $[-1,1]$.

\section{Resultados y discusión}

Clasificación supervisionada del uso del suelo de la cuenca del río Juqueri

Se identificaron las coberturas para la clasificación, además de las líneas base de comparación para validar está. Los resultados de la clasificación se consolidaron en la Figura $x$, de las categorías, de la cobertura por el método de máxima verosimilitud, dando como resultado seis clases: 1) cuerpos de agua - CA; 2) bosque - F; 3) pastoreo - $P$; 4) áreas urbanas - AU; 5) suelo expuesto - SE y 6) áreas agrícolas - AA. (Figura 2) (Anexo).

Se encontró una buena parte del área de la cuenca con problemas de suelos expuestos, específicamente un $11,26 \%$ del área total para el año 2016. Esta clase es muy susceptible a la erosión. Se reconoce que la cuenca hidrográfica del río Juqueri está siendo sometida a una fuerte presión ambiental, pues representa un gran atractivo para el sector inmobiliario, y el aumento en porcentaje en la clase de uso urbano, referente a los años de 2011 hasta 2016 pasó de 13, El 4\% para el 17,51\% del área total de la cuenca (Tabla 5).

Tabla 5. Valores uso del suelo cuenca del rio Juqueri

\begin{tabular}{|l|c|c|c|c|c|c}
\hline Clases \% & $\mathbf{1 9 8}$ & $\mathbf{1 9 9 2}$ & $\mathbf{2 0 0 3}$ & $\mathbf{2 0 1 1}$ & $\mathbf{2 0 1 6}$ & $\mathbf{1 9 8 6}-\mathbf{2 0 1 6}$ \\
\hline Cuerpos de agua & 1,1 & 1,08 & 1,01 & 0,96 & 0,68 & $-0,42$ \\
\hline Bosques & 67,25 & 63,85 & 61,92 & 60,75 & 51,37 & $-15,88$ \\
\hline Pastos & 24,45 & 9,28 & 7,88 & 15,7 & 15,22 & $-9,23$ \\
\hline Áreas Urbanas & 4,02 & 7,19 & 9,99 & 13,04 & 17,51 & $+13,49$ \\
\hline Suelos expuesto & 0,5 & 0,8 & 1 & 2,5 & 3,96 & $+3,46$ \\
\hline Áreas agrícolas & 2,68 & 17,8 & 18,2 & 7,05 & 11,26 & $+8,58$ \\
\hline Total & $\mathbf{1 0 0}$ & $\mathbf{1 0 0}$ & $\mathbf{1 0 0}$ & $\mathbf{1 0 0}$ & $\mathbf{1 0 0}$ & $\mathbf{2 0 1 6}$ \\
\hline Clases ha & $\mathbf{1 9 8 6}$ & $\mathbf{1 9 9 2}$ & $\mathbf{2 0 0 3}$ & $\mathbf{2 0 1 1}$ & $\mathbf{1 9 8 6}-\mathbf{2 0 1 6}$ \\
\hline Cuerpos de agua & 371 & 364 & 341 & 324 & 229 & -142 \\
\hline Bosques & 22673 & 21526 & 20876 & 20481 & 17319 & -5354 \\
\hline Pastos & 8243 & 3129 & 2657 & 5293 & 5131 & -3112 \\
\hline Áreas Urbanas & 1355 & 2424 & 3368 & 4396 & 5903 & 4548 \\
\hline Suelos expuesto & 169 & 270 & 337 & 843 & 1335 & 1167 \\
\hline Áreas agrícolas & 904 & 6001 & 6136 & 2377 & 3796 & 2893 \\
\hline Total & $\mathbf{3 3 7 1 4}$ & $\mathbf{3 3 7 1 4}$ & $\mathbf{3 3 3 1 4}$ & $\mathbf{3 3 7 1 4}$ & $\mathbf{3 3 7 1 4}$ \\
\hline
\end{tabular}

El embalse Paiva Castro que está incluido en los recursos hídricos de la cuenca viene sufriendo una continua degradación, pues su área viene disminuyendo paulatinamente con el tiempo. De acuerdo con los datos obtenidos por esta investigación, la cuenca en comparación de los años 1986 al año 2016 cuenta con menos del $0,42 \%$ de área de los cuerpos de agua.

Cabe resaltar que el embalse es una de las mayores riquezas de la cuenca en la que se 
vienen generando fuertes impactos debido a la reciente construcción de casas de alto padrón en su perímetro.

Su belleza escénica hace también que muchos turistas visiten el embalse y terminen adquiriendo predios, incrementando el área urbana, donde para el año 1986 era del 4,02\% del área total de la cuenca y para el 2016 se incrementó a $17,51 \%$, además de ese crecimiento urbano sin planificación, el área urbana creciente viene acompañada de redes de alcantarillado y aguas residuales sin tratamiento que son liberadas en el embalse en natura.

Se observaron cambios también en las áreas de pastoreo que se disminuyeron en 3.112 ha comparado los años 1986 a los años 2016, donde se percibe que esta disminución fue sustituida por áreas urbanas (4.548 ha) y una pequeña porción por las áreas agrícolas (1.167 ha (Tabla 5).

Se resalta que, al ocurrir la disminución del área de los cuerpos de agua, aumenta la bioacumulación y biodisponibilidad de determinados contaminantes en el embalse Paiva Castro, lo que ocasiona impactos sobre la salud de los seres vivos, incluyendo los invertebrados y las personas usuarias del abastecimiento por este cuerpo hídrico.

La mayor presión ambiental se encuentra en las clases de cuerpos de agua y en las capas forestales de la cuenca del río Juqueri, que a pesar de ser protegida por la Ley de Protección de los Manantiales - Ley Estatal 9,866 / 97, el área forestal que rodea el embalse se está reduciendo (Figura 3)

Se determina que existe gran presión ambiental ocurrida por los cambios en el uso del suelo en la cuenca, donde los mayores impactos fueron ocasionados en el período de los años 2011 hasta 2016, período en el cual se obtuvieron los mayores valores de pérdidas en áreas de clases muy importantes, como la clase de bosque, pastoreo y los cuerpos de agua registrados por las imágenes de satélite. Además, se identificó aumento en los valores de las áreas de las clases de suelo expuesto y para el área urbana, denotando una progresiva degradación ambiental (Figura 2).

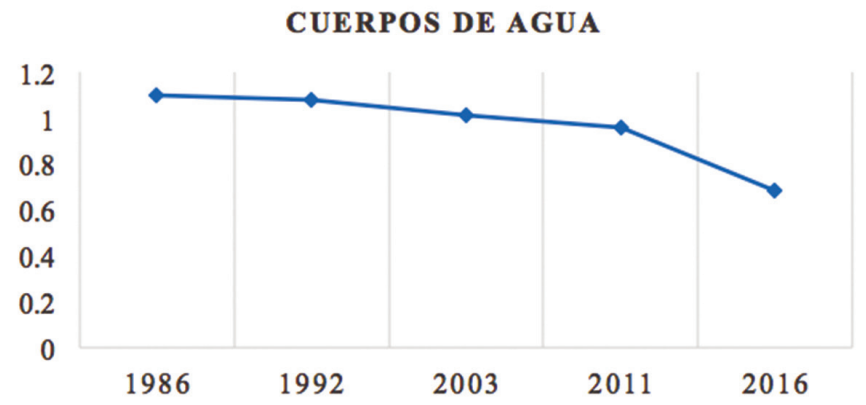

Figura 3. Diminución en \% del área de la clase Cuerpos de agua

\section{Índice de calidad de aguas (ICA)}

En cuanto a la clasificación del agua de la cuenca hidrográfica del río Juqueri según los valores de IQA, los encontrados fueron: el "Regular", que está entre los valores de $(36<51)$, y el "Bueno", que está entre los valores de " $(51<79)$.
El mes de julio tuvo más clasificaciones en el valor regular en el P2 y la semana 1 de julio tuvo siempre la clasificación "Buena" según el ICA. En el mes de diciembre los valores regulares de la clasificación según el ICA fueron más equitativos para todos los puntos, y 
la semana 2 del mes de diciembre fue la que registró todos los valores por encima de 52, que significa una clasificación "Buena" según el ICA. Se observa también que en el mes de julio se obtuvieron un mayor número de muestras con valores de ICA "Regular", con un total de 10 muestras. Para el mes de diciembre sólo se obtuvieron 8 muestras con el ICA medio clasificado como "Regular", esto aumentando de 5 a 7 valores totales del ICA de muestra clasificadas como "Buena" (Tabla 6).

Esto podría significar, que el período de lluvia afecta de manera positiva al ICA de la cuenca del río Juqueri, pero el oxígeno disuelto disminuye en este período, al igual que el $\mathrm{pH}$, el Nitrógeno total y el fosforo total. Los sólidos totales en el
P1 aumentan, pero en el P2 y P3 disminuyen su valor. En cuanto al resto de parámetros como la temperatura, los coliformes termotolerantes, DBO y la turbidez, éstos aumentan sus valores en el mes de diciembre. De esta forma, se concluye que existe una dinámica referente a los períodos de precipitación, pues, se puede afirmar que en el caso de la cuenca hidrografía del río Juqueri algunos parámetros aumentan en este período y otros disminuyen en sus contenidos. Los mayores valores de ICA se encontraron en el P3, y los menores valores en P2 y P1. Cabe resaltar que los comportamientos del ICA en los puntos de la salida de agua y en el medio de la cuenca, evidencian una degradación paulatina al transcurso del río en la cuenca por las actividades antrópicas ejercidas en ésta.

Tabla 6. Valores obtenidos de ICA del agua de la Cuenca del río Juqueri en el año 2016.

\begin{tabular}{|c|c|c|c|c|c|c|c|}
\hline Fechas/Julio & P1 & P2 & P3 & Fechas/Diciembre & P1 & $\mathbf{P 2}$ & P3 \\
\hline 1 & 52 & 55 & 52 & 2 & 56 & 52 & 50 \\
\hline 9 & 51 & 45 & 51 & 9 & 55 & 54 & 56 \\
\hline 16 & 39 & 38 & 54 & 16 & 49 & 47 & 51 \\
\hline 30 & 46 & 50 & 51 & 21 & 50 & 46 & 49 \\
\hline Promedio & 47 & 47 & 52 & Promedio & 52.5 & 49.75 & 51.5 \\
\hline Promedio mes & & & 48,6 & & \multicolumn{2}{|c|}{ Media mes } & 51,25 \\
\hline \multicolumn{7}{|c|}{ Media General de Muestras } & 49,95 \\
\hline
\end{tabular}

En color amarillo se clasifica el agua como Regular, en color verde se clasifica el agua como Buena, según la CONAMA 357/2005 de la Ley brasilera. Según la clasificación colombiana propuesta por Fernández \& Solano (2005), el color amarillo se clasifica como agua de calidad media y el color verde como agua de calidad Buena.

Un fenómeno se dio en el P3, pues existe un ligero equilibrio en el embalse Paiva Castro, ya que éste mantiene cierta estabilidad, sobre el valor del ICA 50 (con excepción de la muestra del día 21 que llegó a 49). Esta estabilidad con altos valores de ICA indica que el embalse mantiene generalmente una buena calidad de agua y posee una gran capacidad de depurar los recursos hídricos que llegan a su perímetro.

\section{Correlaciones canónicas entre ICA y uso del suelo de la Cuenca del río Juqueri Correlaciones Canónicas entre los pará- metros del ICA y el uso del suelo de la cuenca hidrográfica del río Juqueri.}

En la Tabla 7 se insertan los valores de la desviación estándar, éste indica una medida de dispersión de los datos en torno a media muestral. El mayor valor se obtuvo en la variable de coliforme termotolerantes con un 4447,2 siendo un 
valor alto que indica que los valores en los puntos muestreados están esparcidos por una amplia gama de valores y muestra contaminación por coliformes con altos valores en esta variable.
Las variables restantes obtuvieron valores relativamente bajos, indicando que los valores en los puntos muestreado tienden a estar más cerca de la media e indican cierta estabilidad en estos.

Tabla 7. Estadística descritiva de lós parametros ICA

\begin{tabular}{l|c|c|c|c|c|c|c|c|c|c|c|c|}
\hline & OD & T & Coliformes & PH & DBO & NT & P & Turb, & ST & IQA \\
\hline Promedio & 5,6 & 23,2 & 3411,3 & 6,6 & 4,2 & 1,8 & 0,4 & 4,0 & 0,0 & 50,1 \\
\hline Desvió Padron & 1,2 & 3,7 & 4447,2 & 0,6 & 1,4 & 1,8 & 0,6 & 1,8 & 0,0 & 2,8 \\
\hline Mínimo & 4,0 & 18,4 & 94,7 & 5,8 & 1,0 & 0,4 & 0,2 & 1,8 & 0,0 & 43,7 \\
\hline Máximo & 9,4 & 28,8 & 13745,3 & 8,3 & 6,1 & 7,1 & 2,7 & 7,8 & 0,1 & 55,0 \\
\hline Coeficiente Variación & 0,2 & 0,2 & 1,3 & 0,1 & 0,3 & 1,0 & 1,6 & 0,4 & 1,3 & 0,1 \\
\hline
\end{tabular}

Las coliformes presentaron mayor promedio, desviación estándar y coeficiente de variación mientras que el pH mostró menor variabilidad (Tabla 7). Estos valores representan la totalidad de las estaciones muestreadas en la cuenca hidrográfica del río Juqueri. Los coeficientes de variación relativamente altos son indicación de la inconstancia de los valores de los parámetros a lo largo del tiempo y a lo largo de la line salida aguas abajo, influyendo de manera relevante en el ICA. Evidentemente, como consecuencia de las actividades antrópicas en el área de estudio, esta gran variabilidad se espera.

En referencia a la matriz de correlación, ésta posibilitó identificar las variables más significativas, que cumplen un papel importante en la interpretación de los datos del estudio. La dependencia de las variables medidas puede ser vista por medio de la matriz de correlación (Tabla 8).

Tabla 8. Matriz de correlación ACP de los parámetros del ICA y los usos del suelo

\begin{tabular}{|c|c|c|c|c|c|c|c|c|c|c|c|c|c|c|c|c|}
\hline & OD & $\mathbf{T}$ & COL & pH & DBO & NT & PT & TUR & ST & IQA & CA & $\mathbf{F}$ & $\mathbf{P}$ & $A U$ & SE & AA \\
\hline OD & 1,0 & & & & & & & & & & & & & & & \\
\hline $\mathrm{T}$ & 0,6 & 1,0 & & & & & & & & & & & & & & \\
\hline $\mathrm{COL}$ & $-0,4$ & $-0,4$ & 1,0 & & & & & & & & & & & & & \\
\hline $\mathrm{pH}$ & $-0,5$ & $-0,6$ & 0,9 & 1,0 & & & & & & & & & & & & \\
\hline DBO & $-0,2$ & 0,2 & 0,2 & 0,3 & 1,0 & & & & & & & & & & & \\
\hline NT & $-0,0$ & $-0,3$ & $-0,3$ & $-0,4$ & $-0,8$ & 1,0 & & & & & & & & & & \\
\hline PT & $-0,3$ & $-0,5$ & $-0,2$ & 0,1 & 0,1 & 0,0 & 1,0 & & & & & & & & & \\
\hline TUR & $-0,6$ & $-0,8$ & 0,8 & 0,9 & 0,0 & $-0,0$ & 0,3 & 1,0 & & & & & & & & \\
\hline ST & $-0,3$ & $-0,2$ & $-0,5$ & $-0,5$ & $-0,5$ & 0,8 & 0,1 & $-0,1$ & 1,0 & & & & & & & \\
\hline IQA & 0,5 & 0,8 & 0,0 & $-0,3$ & 0,2 & $-0,3$ & $-0,8$ & $-0,5$ & $-0,3$ & 1,0 & & & & & & \\
\hline$C A$ & $-0,8$ & $-0,8$ & 0,2 & 0,3 & $-0,1$ & 0,4 & 0,3 & 0,6 & 0,5 & $-0,7$ & 1,0 & & & & & \\
\hline $\mathrm{F}$ & $-0,4$ & 0,0 & $-0,3$ & $-0,1$ & 0,6 & $-0,2$ & 0,6 & $-0,1$ & 0,2 & $-0,3$ & 0,2 & 1,0 & & & & \\
\hline $\mathrm{P}$ & 0,8 & 0,8 & $-0,6$ & $-0,7$ & 0,0 & $-0,1$ & $-0,3$ & $-0,9$ & -0 & 0,6 & $-0,8$ & $-0,0$ & 1,0 & & & \\
\hline$A U$ & $-0,7$ & $-0,0$ & 0,3 & 0,3 & 0,7 & $-0,4$ & $-0,0$ & 0,2 & 0,0 & 0,0 & 0,3 & 0,5 & $-0,4$ & 1,0 & & \\
\hline SE & $-0,0$ & 0,6 & $-0,2$ & $-0,3$ & 0,5 & $-0,3$ & $-0,3$ & $-0,5$ & 0,1 & 0,5 & $-0,2$ & 0,5 & 0,3 & 0,6 & 1,0 & \\
\hline$A A$ & $-0,2$ & 0,5 & $-0,0$ & $-0,1$ & 0,6 & $-0,4$ & $-0,3$ & $-0,3$ & 0,0 & 0,4 & $-0,0$ & 0,4 & 0,1 & 0,8 & 0,9 & 1,0 \\
\hline
\end{tabular}


Los resultados de las correlaciones identificadas entre parámetros del ICA fueron ( $T$ - OD con valor 0,6$),(\mathrm{pH}$ - Coliformes con valor 0,9$)$, (Turb - pH con valor 0,9 ) siendo estas dos últimas las de mayor valor de correlación, ( $T$ - IQA con valor 0,8$)$ y $($ ST - NT con valor 0,8$)$.

En cuanto a las correlaciones canónicas la correlación que presentó los mayores coeficientes de correlaciones fue a (áreas Pasto - $T$ con un valor 0,8 ) (Suelo expuesto - $T$ con un valor de 0,6$)$, esto denota una dependencia de los valores de la temperatura en cuanto a las temperaturas áreas de pastoreo y áreas de suelo expuesto contenidos en la cuenca (Tabla 8).

Al aumentar las áreas de pastoreo y suelos expuestos, disminuyen las áreas de bosque. La energía del sol entra directamente sobre el suelo y los recursos hídricos disminuyen por el aumento de la evapotranspiración, lo que ratifica la correlación entre la temperatura con las áreas de pastoreo y los suelos expuestos que viene aumentando en los últimos 30 años.

\section{Correlación entre la DBO y las áreas urbanas}

En la Tabla 8 y en las Figuras 4 y 5 se identifican las correlaciones entre DBO y el área urbana con un valor de 0,7 , donde generalmente las aguas del alcantarillado de una ciudad se componen de desechos domésticos e industriales donde el parámetro más utilizado para clasificar si el alcantarillado es sanitario o industrial es la DBO, cuanto mayor sea DBO, mayor es la contaminación orgánica lo que podría explicar la correlación entre estas variables (Ferreira 2010).

Este autor también afirma que la composición del desagüe es bastante variable, presentando mayor contenido de impurezas durante el día y menor durante la noche. La materia orgánica, especialmente las heces humanas, confiere al alcantarillado sanitario sus principales características, cambiantes con el transcurrir del tiempo, pues sufre diversas alteraciones hasta su completa mineralización o estabilización.

Mientras que el desagüe sanitario causa contaminación orgánica y bacteriológica, el industrial generalmente produce la contaminación química.

El efluente industrial, además de las sustancias presentes en el agua de origen, contiene impurezas orgánicas y/o inorgánicas resultantes de las actividades industriales, en cantidad y calidad variables con el tipo de industria. Los cuerpos de agua pueden recuperarse de la contaminación, o depurarse por la acción de la propia naturaleza. El efluente generalmente puede ser lanzado sin tratamiento en un curso de agua, siempre que la descarga contaminante no sobrepase cerca de cuarenta avos del caudal: un río con 120 L/s de caudal puede recibir de grosso modo la descarga de $3 \mathrm{~L} / \mathrm{s}$ de flujo de acuerdo con la normativa vigente.

Según el PLAN MUNICIPAL DE SANEAMIENTO BÁSICO DE MAIRIPOR, los servicios de agua y alcantarillado son prestados por la Compañía de Saneamiento Básico del Estado de São Paulo SABESP, a través de un contrato fechado de más de 30 años atrás y que tuvo su validez expirada en 2009. En los últimos 10 años, poco se ha hecho para Universalización del acceso al agua de abastecimiento, para garantizar la calidad del manantial y los recursos hídricos de la cuenca en cuestión y de asegurar las condiciones sanitarias de la población. De acuerdo con los censos de 2000 y 2010 realizados por el IBGE, la parcela de domicilios particulares permanentes del municipio con acceso a la red de abastecimiento pasó de sólo el $65 \%$ al $71 \%$. La red de recolección de aguas residuales a su vez pasó solamente del $31 \%$ al $35 \%$ en el mismo período (IBGE 2012).

De este modo se concluye que más de la mitad de las alcantarillas generadas están dispuestas en 
fosas o arroyos en natura. La insuficiencia del sistema colector existente viene comprometiendo la calidad de las aguas superficiales de la cuenca, así como de su propio manantial de abastecimiento, una vez que el efluente es lanzado desde el punto de captación, causando condiciones sanitarias insalubres. Esta realidad corrobora la correlación existente entre la DBO y el Área Urbana, pues ésta viene creciendo a través del tiempo sin una planificación previa y más del 35\% de las áreas urbanas del Municipio de Mairiporã no tiene red de recolección de aguas residuales, lo que intensifica la contaminación por materia orgánica y hace incrementar los valores de la DBO (Figura 4).

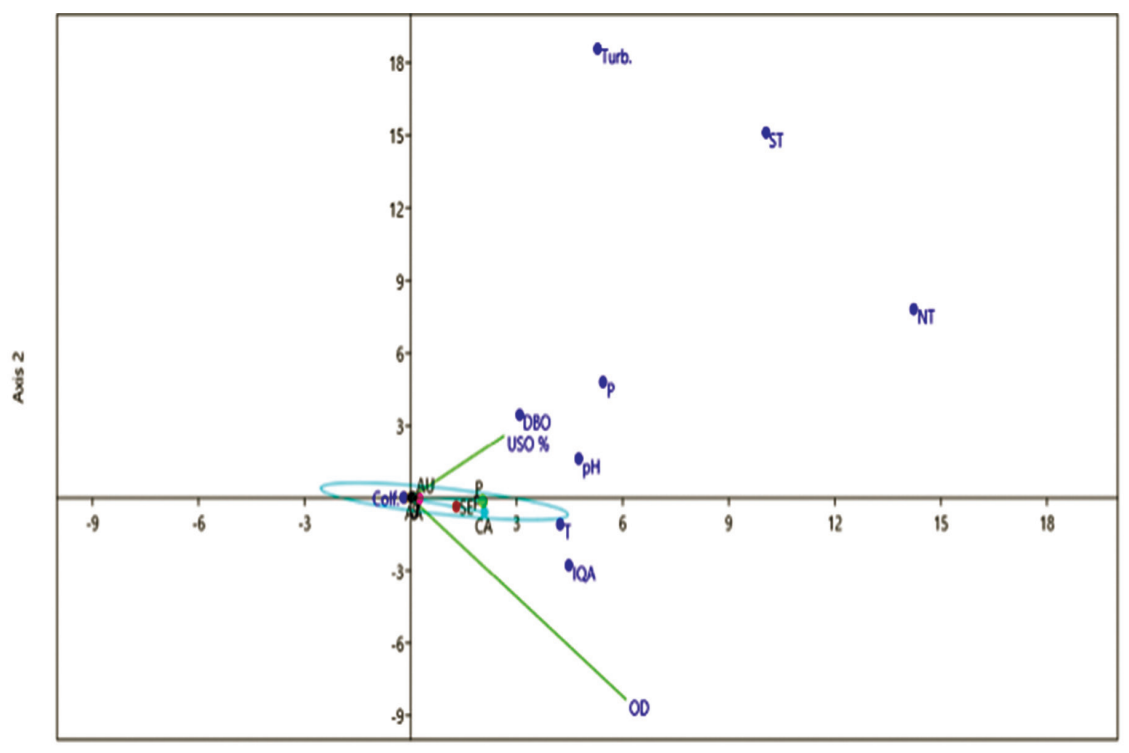

Avis 1

Figura 4. Correlaciones canónicas de las variables de uso de suelos y los parámetros del ICA de los ejes 1 y 2 . Fuente: esta investigación

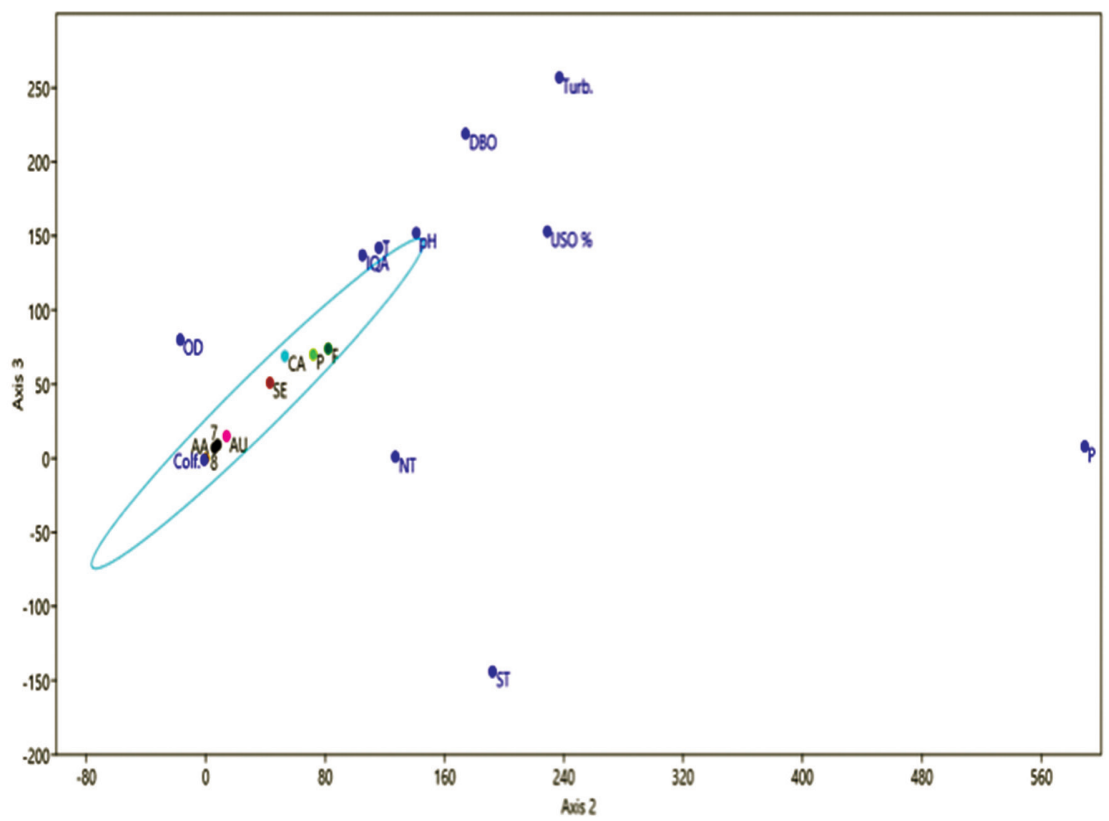

Figura 5. Correlaciones canónicas de las variables de uso de suelos y los parámetros del ICA de los ejes 2 y 3 Fuente: Esta investigación 


\section{Correlación entre la Turbidez y las áreas de los Cuerpos de agua}

La Tabla 8 y las Figuras 4 y 5, también demostraron con un valor de 0,6 la correlación entre la turbidez con el área de los cuerpos de agua, los mayores valores de turbidez en la cuenca hidrográfica del río Juqueri se encontraron en el P2, punto que se encuentra al lado del área urbana en la cuenca.

Los autores Menezes, et al., (2016), encontraron en la cuenca hidrográfica urbana del Ribeirão Rojo, que las cargas de sólidos en el agua y la turbidez, eran mayores en los puntos muestreados que eran secciones con fuerte ocupación agrícola y urbana.

Esto corrobora las correlaciones entre las áreas urbanas y cómo estas modifican y empeoran los valores encontrados de turbidez tanto en esta investigación, cuando en la cuenca hidrográfica urbana del Ribeirão Vermelho.

Es bueno entender que la turbidez es una medida del grado en que el agua pierde su transparencia debido a la presencia de partículas en suspensión (presencia de fitoplancton o crecimiento de algas, sedimentos provenientes de la erosión, y el lanzamiento de efluentes, tales como el agua del flujo urbano mezclado).

A continuación se describen todas las correlaciones canónicas encontradas en la cuenca hidrográfica del río Juqueri obtenidas en las Figuras 4 y 5 (Tabla 9).

Tabla 9. Correlaciones canónicas Cuenca del río Juqueri

\begin{tabular}{|c|c|c|c|}
\hline $\mathbf{N}^{0}$ & Matriz correlación & Eje 1 e 2 & Eje 3 e 4 \\
\hline 1 & $\begin{array}{l}\text { *Temperatura - } \\
\text { área de Pastos. }\end{array}$ & **Coliformes - área Urbana. & $\begin{array}{l}\text { **Coliformes - área Urbana } \\
\text { y Área Agrícola. }\end{array}$ \\
\hline 2 & $\begin{array}{l}\text { *Temperatura - área } \\
\text { de Suelo expuesto. }\end{array}$ & $\begin{array}{l}\text { *Temperatura - pastagem e } \\
\text { Solo exposto. }\end{array}$ & $\begin{array}{l}\text { *Temperatura - área da pastos y área de Bosque } \\
\text { (influenciada por todos los usos de suelo) }\end{array}$ \\
\hline 3 & DBO - Área Urbana & $\begin{array}{l}\text { Correlaciona dos ST e NT } \\
\text { - Turbidez, (afastados dos } \\
\text { outros parametros) }\end{array}$ & Correlación Turbidez - DBO \\
\hline 4 & DBO - Área Agrícola & $\begin{array}{l}* * * \text { Correlación todos los } \\
\text { usos - Coliformes } \\
\text { y Temperatura. }\end{array}$ & $\begin{array}{l}* * * \text { Correlación Todos los usos - } \\
\text { Coliformes, Temperatura, } \mathrm{pH} \text { y ICA }\end{array}$ \\
\hline 5 & $\begin{array}{l}\text { Turbidez - Área } \\
\text { de cuerpos de agua }\end{array}$ & & Correlación ST - NT y el PT \\
\hline 6 & PT - Bosque & & \\
\hline 7 & ICA - Área de pasto & & \\
\hline
\end{tabular}

Una variable que puede afectar mucho a los ecosistemas acuáticos es la turbidez, pues deteriora el proceso de fotosíntesis limitando el paso de la luz solar, la respiración y la reproducción de la vida acuática, donde las partículas en suspensión absorben el calor del sol, tornando las aguas más turbias y más calientes, y reduciendo, así, la concentración de 
oxígeno en el agua, disminuyendo la actividad fotosintética en las algas, lo que contribuye para disminuir aún más a concentración de oxigeno (CETESB, 2014).

Esta sería una correlación directa con la OD y la DBO, siendo así, en este caso podemos afirmar que en los períodos lluviosos los aumentos de cantidad de agua precipitada hacen lavado de las áreas que limitan el perímetro de los arroyos, ríos y del embalse Paiva Castro, llevando consigo material coloidal y suelo erosionado, lo que explicaría la correlación obtenida entre la turbidez y la clase de cuerpos de agua.

\section{Correlación entre el Fósforo total y las áreas de Bosque}

En las Figuras 4 y 5 y la Tabla 9, se identifican las correlaciones entre el fosforo y las áreas forestales. Ahora, una posible explicación para esta correlación es dada por Malavolta (1996), que afirma que los bosques desempeñan un papel importantísimo sobre el ciclo de carbono, los nutrientes minerales con el fósforo y la retención de agua en el suelo, lo que explicaría esta correlación.

El mismo autor afirma que existe una correlación directa entre las áreas con cobertura forestal (vegetación secundaria) y el $\mathrm{P}$, debido a simbiosis de sus raíces con micorrizas, que en el período de lluvias sea por erosión de suelos o por flujo subterráneo el fósforo está disponible en los recursos hídricos de la cuenca hidrográfica del río Juqueri en pequeñas cantidades, también hay que decir que las mayores fuentes de fósforo son de fertilizantes hechos a base de éste y en las aguas residuales urbanas no tratadas.

Además, en un estudio en el embalse de Itupararanga, São Paulo, Cunha y Calijuri (2011) encontraron concentraciones de fósforo cercanas a las determinadas en ese trabajo y concluyeron a partir de observaciones de datos de 16 reservorios brasileños que hay correlación positiva entre las concentraciones de fósforo total y clorofila en cuencas hidrográficas con uso del suelo predominantemente agrícola y forestal.

Existe una baja disponibilidad de nutrientes, principalmente de fósforo en los suelos de São Paulo y puede limitar el crecimiento de la vegetación secundaria. Sin embargo, los bosques tropicales presentan estrategias para maximizar la absorción de nutrientes, como asociaciones con hongos micorrícicos y producción de raíces apogeotrópicas, donde los bosques secundarios generalmente logran desarrollarse en suelos con deficiencia de fósforo posiblemente en parte debido al establecimiento de simbiosis de micorrizas y las raíces (Coelho y Verlengia, 1993).

Diversos factores ambientales pueden influenciar la colonización micorrícica y el crecimiento radicular en ecosistemas forestales, tales como disponibilidad de nutrientes y agua en el suelo, además de cambios en la cobertura del suelo (Malavolta, 1996).

\section{Correlación entre el ICA y las Pastos}

En las correlaciones encontradas en las Figuras 4 y 5 y plasmada en la Tabla 9, entre el ICA y las áreas de pastoreo, se encontró una similitud en el trabajo realizado por el autor Fia et al., (2015), el cual encuentro en la cuenca hidrográfica del Ribeirão Rojo, a lo largo del río y de sus afluentes, valores de ICA bajos, y de calidad entre media a mala, y que estos valores eran resultado del área urbana del municipio de Lavras. Sin embargo, el mismo autor vio que existía un comportamiento de depuración de la calidad de sus aguas pasando por áreas ocupadas por áreas de bosque y pastos con vegetación secundaria. El autor afirma que los 
valores de ICA que obtuvo no tuvieron niveles aceptables para el consumo, pero si encontró mejoras en los ICA mediante la autodepuración de los recursos hídricos. este fenómeno podría explicar las correlaciones entre el ICA y las áreas de pastoreo.

\section{Conclusiones}

El aumento poblacional del área urbana, acompañada de diversas interferencias humanas ligadas al mal uso del espacio urbano, incrementos de las áreas de suelos expuestos que son susceptibles a cualquier tipo de erosión, además de una disminución de bosques y degradación de sus recursos hídricos en cantidad y calidad, son problemas con los que la cuenca del río Juqueri necesita trabajar para reducir en cierto modo la fuerte presión ambiental en la que se encuentra sometida.

EI ICA es una fuente de información importante sobre el estado de calidad del agua de la cuenca, pues clasificó sus recursos hídricos dando como resultado promedio de 49,28 clasificando el agua de la cuenca como Regular. Recordando que esta cuenca contiene un manantial importantísimo, el cual ofrece agua a 19 millones de personas en la ciudad de São Paulo. Esto deja claro que se necesita urgentemente una gestión de la cuenca para mejorar estos valores, recordando que sus recursos hídricos están clasificados como Clase I, según la legislación estadual paulista referente a los recursos hídricos superficiales (ley no 997 76 , regulada por el decreto 8.46876 ), siendo estas aguas destinadas al abastecimiento doméstico, determinando que cualquier tipo de lanzamiento de aguas negras, incluso aguas tratadas está prohibido en los cuerpos hídricos de esta categoría (CETESB, 2014).

Los altos valores de la DBO sobrepasaron el valor estándar según la CONAMA 357/2005 que clasifica los cuerpos de agua y las directrices ambientales para su encuadramiento. Esto implica una mayor cantidad de materia orgánica proveniente del área urbana, está siendo degradada por los microorganismos, que hacen disminuir la cantidad de oxígeno para las especies aerobias de los ecosistemas acuáticos, elevando la demanda del oxígeno disuelto, demostrando la existencia de la correlación entre la DBO y el pH y la DBO con las áreas urbanas.

Las correlaciones canónicas se mostraron muy útiles para determinar la presión y degradación de los recurso hídricos de la cuenca hidrográfica del río Juqueri, pues mediante estas fue posible simplificar y determinar que usos del suelo están impactando más la cuenca, además se verifico que las áreas urbanas y agrícolas influencian en la reducción de los valores de variables del ICA como la DBO y el oxígeno disuelto, además de aumentar los valores en otras como la turbidez, sólidos totales, nitrógeno y fosforo. Estas variables son muy importantes y están causando mayores impactos en los recursos hídricos, siendo así, se concluye las correlaciones canónicas ayudaron significativamente en la reducción de la complejidad y consecuentemente en la facilidad de la interpretación de los problemas ambientales en los recursos hídricos de la cuenca.

Se constataron correlaciones importantes entre la disminución en los valores de DBO por las áreas urbanas, y mejoras en los valores de ICA por las áreas de Pastoreo. Ante lo expuesto, se concluye que existe una necesidad urgente de planificación del uso y ocupación de suelos en el área de la cuenca.

\section{Literatura citada}

BRASIL. [homepage on the internet]. Dispõe sobre a classificação dos corpos de água e diretrizes ambientais para o seu enquadramento, bem como estabelece as condições e padrões de lançamento de efluentes, e dá outras providências: Resolução no 357 do Conselho Nacional de 
Meio Ambiente. [update 2005 Mar 18; cited 2017 Abril 10]. Available from: http://www.mma.gov. br/port/conama/res/res05/res35705.pdf

CETESB Companhia Ambiental Do Estado De São Paulo - [homepage on the internet]. Índice de qualidade das águas - IQA. São Paulo. [update 2007 Fev 21; cited 2016 Dec 11]. Available from: http://portalpnqa.ana.gov.br/indicadores-indiceaguas.aspx

COELHO, F.S. \& VERLENGIA, F 1993. Fertilidade do solo. Campinas, Instituto Campineiro de ensino agrícola. 384p.

Cunha DF, Calijuri MD 2011. Limiting factors for phytoplankton growth in subtropical res ervoirs: the effect of light and nutrient avail ability in different longitudinal compart ments. Lake Reserv. Manag. 27: 162-172.

DOWNING, J.A. 2004. A Manual on Methods for the Assessment of Secondary Productivity in Fresh Waters. Blackwell Scientific Publications, Oxford, England.

FERREIRA W. Estudo de patógenos e metais em lodo digerido bruto e higienizado para fins agrícolas, das estações de tratamento de esgotos da ilha do governador e da Penha no estado do Rio de Janeiro. [Dissertação de Mestrado]. Rio de Janeiro: Fundação Oswaldo Cruz, Escola Nacional de Saúde Pública, 2010 [citado o 2016 Nov 10]. Disponível em: https:// www.researchgate.net/publication/36168803_Estudo_de_patogenos_e_metais_em_lodo_digerido_bruto_e_higienizado_para_fins_agricolas_ das_estacoes_de_tratamento_de_esgotos_da_ Ilha_do_Governador_e_da_Penha_no_Estado_ do_Rio_de_Janeiro_electronic_reso.

HADDAD, E. Influências antrópica na qualidade da água da bacia hidrográfica do rio São Miguel, Carste do alto São Francisco, Minas Gerais. 2007. 156 f. Dissertação (Mestrado em Geografia) Programa de pós-graduação em geografia, Universidade Federal de Minas Gerais - UFMG, Belo Horizonte, 2007.
IBGE Instituto Brasileiro De Geografia E Estatística 2012. Manual Técnico da Vegetação Brasileira, IBGE, Rio de Janeiro.

IDEAM 2010. Leyenda nacional de coberturas de la tierra, Metodología CORINE Land Cover adaptada para Colombia escala 1:100.000. Instituto de Hidrología, Meteorología y Estudios Ambientales.

MALAVOLTA, E 1996. Manual de química agrícola: Nutrição de plantas e fertilidade do solo. São Paulo, Editora Agronômica Ceres. São Paulo. 528 p.

MENEZES J, BITTENCOURT R, FARIAS M, BELLO I, FIA R, COUTINHO L 2016. Relação entre padrões de uso e ocupação do solo e qualidade da água em uma bacia hidrográfica urbana. Revista de Engenharia Sanitária e Ambiental. Vol.21 n.3. p 519-534.

OLIVEIRA-FILHO PC, DUTRA AM, CERUTI FC 2012. Qualidade das águas superficiais e o uso da terra: estudo de caso pontual em bacia hidrográfica do oeste do Paraná. Revista Floresta e Ambiente, Seropédica, v.19, v.1

PÉREZ-ORTEGA DJ, PÉREZ D, AMÉRICO J, CARVALHO S, SEGOVIA J 2016. Development of Index of Resilience for Surface Water in Watersheds. Journal of Urban and Environmental Engineering, v.10, n.1, p.72-82.

POLETO, C. Monitoramento e avaliação da qualidade da água de uma microbacia hidrográfica no município de ilha solteira - s.p. 2003. 150 f. 143 Dissertação (Mestrado) - Faculdade de Engenharia Civil na área de recursos hídricos e tecnologias ambientais, Universidade Estadual Paulista - UNESP, Ilha Solteira, 2003.

RIBEIRO KH. Qualidade da água superficial e a relação com o uso do solo e componentes ambientais na microbacia do rio Campestre, [Dissertação de Mestrado] Colombo. Universidade Federal do Paraná, 2009. [citado o 2016 Nov 10]. Disponível em:http://www.scielo.br/scielo. php?pid $=$ S010006832014000200030\&script $=s$ ci_abstract\&tIng=pt. 
